\title{
Improved AODV route recovery in mobile ad-hoc networks using a genetic algorithm
}

\author{
Ahmad Maleki $^{\mathbf{a}^{*}}$ and Mehdi Sadeghzadeh ${ }^{\mathrm{b}}$
}

${ }^{a}$ Department of Computer Engineering, Damavand Science and Research Branch, Islamic Azad University, Damavand, Iran ${ }^{b}$ Department of Computer Engineering, Mahshahr branch, Islamic Azad University, Mahshahr, Iran

\section{H R O N I C L E}

Article history:

Received March 22014

Accepted 29 July 2014

Available online

July 292014

Keywords:

Mobile ad-hoc networks

AODV

Route recovery mechanisms

Genetic algorithm

NS-2 simulator

\section{A B S T R A C T}

An important issue in ad-hoc on-demand distance vector (AODV) routing protocols is route failure caused by node mobility in the MANETs. The AODV requires a new route discovery procedure whenever a route breaks and these frequent route discoveries increase transmission delays and routing overhead. The present study proposes a new method for AODVs using a genetic algorithm to improve the route recovery mechanism. When failure occurs in a route, the proposed method (GAAODV) makes decisions regarding the QOS parameter to select source or local repair. The task of the genetic algorithm is to find an appropriate combination of weights to optimize end-to-end delay. This paper evaluates the metrics of routing overhead, average end-to-end delay, and packet delivery ratio. Comparison of the new algorithm and AODV (RFC 3561) using a NS-2 simulator shows that GAAODV obtains better results for the QOS parameters.

\section{Introduction}

Wireless technology has now reached the stage where communication is possible at any point on the earth. Millions of people transmit and exchange information using technologies such as mobile phones, personal digital assistants and other wireless devices. Mobile ad-hoc networks (MANETs) are a set of distribution nodes without the need for intervention of a centralized control or existing infrastructure (Sarkar et al., 2007). Each node in a MANET is equipped with a wireless receiver and a transmitter and can act as both a host and router to forward packets to other existing nodes. The most essential feature of these networks is the existence of a dynamic and variable topology resulting from node mobility. Nodes in this network continuously change position. This needs a routing protocol that has the ability to adapt to these changes (Al-Omari \& Sumari, 2010). The Internet Engineering Task Force has suggested many routing protocols. These protocols can be grouped into position based (GPSR, GEOTORA, GPCR) and topology based (reactive; AODV, DSR, proactive; DSDV, OLSR, hybrid; ZRP). In proactive protocols, all nodes have consistent and up-to-date permanent routing *Corresponding author.

E-mail addresses: malekiahmad@yahoo.com (A. Maleki) 
information for each node. Reactive protocols, by contrast, generate routes when demanded by the source node (Mbarushimana \& Shahrabi, 2007). Hybrid protocols combine the advantages of proactive and reactive routing. In the AODV protocol, route discovery begins only when the source node intends to communicate with a destination node (Perkins \& Royer, 1999). When a failure happens in a link, the upstream node informs the source node that its connection with the destination has interrupted by a RERR message. If the distance from the broken link to the destination node is less than the distance to the source node, local routing is implemented. Otherwise, rerouting begins again from the source node, which decreases the packet transmission delay and the amount of control routing.

The proposed method applies a genetic algorithm (GA) for routing and considers the neighbors of the candidate node. The task of the genetic algorithm is to determine an appropriate combination of weights to optimize the fitness function (average end-to-end delay) to repair the AODV route. The proposed method improves the AODV (RFC 3561 standard) using the GA method. The results of simulation to validate the proposed method indicate substantial improvement in the packet delivery ratio, overhead and average end-to-end delays. Section 2 of this paper introduces the AODV routing protocol, route recovery mechanisms, and reviews related work. Section 3 describes the proposed GA ad-hoc on-demand distance vector (GAAODV). Simulation results are given in Section 4. Section 5 presents the main conclusions of the research and formulates the main avenues for future study.

\section{AODV Route Recovery Mechanisms}

Perkins and Royer are believed to be the first who presented the AODV as a reactive on-demand routing protocol for ad-hoc mobile networks. It was an improvement on the destination sequenced distance vector (DSDV) because it minimizes the number of broadcasts required by creating routes on a demand basis (Rahman \& Zukarnain, 2009). According to RFC 3561 standards (Das et al., 2003), a node wishing to communicate with another specific node first searches for a route in its routing table. If it finds one, the communication to destination starts without any delay; otherwise the node initiates a route discovery stage. The route discovery procedure consists of broadcasting a routerequest message (RREQ). If a node maintains a valid route to the destination node, it gives a feedback to the RREQ with a route-reply message (RREP). The replying node generates a reverse route entry in its routing table that contains the number of hops to the source, the next hop's address, and the address of the source node. In AODV, a node may provide connectivity information by broadcasting hello messages. Each node periodically broadcasts hello messages to inform its neighbors that it has not moved away.

In the route maintenance phase, when a node detects a link failure, it creates a route error message (RERR). This RERR is propagated over routes and simultaneously invalidates the corresponding routes. When a RERR is sent back to a source node, the source node initiates a new route discovery procedure. Alternatively, the node upstream of that break may choose to repair the link locally if the destination is not too far away. It initiates the repair, and then waits for the discovery period to receive a RREP in response to the RREQ.

\subsection{Related work}

The need to provide efficient communications in mobile ad-hoc networks has motivated the research community to address the problem of path failure in the AODV routing protocol. Studies have concentrated on preparing dramatic improvements in the quality of service (QOS), such as for the packet delivery ratio, average end-to-end delay, and overhead. Youn et al. (2006) presented a promiscuous mode-based quick local repair technique, which decreases the energy overhead for the lifetime of the network. If there is chance to participate in the repair of link failure, then the node works in promiscuous mode; however, it is not always possible to repair the link failure locally. Dong 
et al. (2011) suggested a recovery mechanism using autonomous underwater vehicles (AUVs) based on an ad-hoc on-demand distance vector. This process employs the hello message applied in route maintenance. Pan et al. (2005) introduced a local repair method based on breadth and depth route repair request constraints. This approach applies bonus gain, which is the ratio of the throughput increment to the routing overhead increment. Bilgin and Khan (2010) proposed the shrink procedure to dynamically optimize routing that does not require restarting the route discovery. The shrink procedure is activated at a certain probability by sending a message through the RSS to detect whether there is redundancy in the routing path and optimal path. Other researchers such as Marina and Das (2011), Liu and Raychaudhuri (2005), Huang et al. (2010) and Yujun and Lincheng (2010) used multipath routing to improve the performance of AODV protocol. Multipath routing increases network resilience to route failure. Multiple paths are either end-to-end disjoint paths or braided paths. For end-to-end disjoint paths, multiple paths are built between the source and destination nodes where the backup paths do not intersect the primary path.

\section{Genetic algorithm ad hoc on-demand distance vector (GAAODV) method}

The proposed GAAODV method of this paper acts as a conventional AODV protocol to choose source repair or local repair at the time of link failure. The difference in this new method is that it considers the number of existing neighbors in the node transmission area. The methodology applied in this study is to explore the search space for possible combinations of four parameter values using a genetic algorithm (GA).

\subsection{Genetic Algorithm}

Evolutionary algorithms (EA) are nondeterministic methods that emulate the evolutionary process in nature to solve optimization, search, and other complex problems. The genetic algorithm (GA) is an evolutionary algorithm, which involves processes such as initialization, selection, mutation, crossover and termination (Gen \& Cheng, 2000). In a GA, a population of strings (the genotype of the genome or chromosomes) that encode candidate solutions to an optimization issue evolves toward better solutions. The GA proposed by Holland (Whitley, 1994) is derived from natural selection and natural genetics. It maintains a population of creatures for a generation. Each creature represents a potential solution to the problem and is evaluated to give some measure of fitness. Some creatures undergo random transformations by means of genetic operations and form new creatures. There are two main genetic operators of transformation. Crossover forms a new creature by combining parts from two other creatures. Mutation forms a new creature by changing a single creature. A new population is formed by selecting the more fit creatures from the parent population and the transformed offspring population. After several generations, the GA converges to the best creature, which represents an optimal or suboptimal solution to the problem. The stopping criterion usually involves a fixed number of generations or execution times, the detection of stagnation, or a quality threshold for the best fitness value.

\subsection{GAAODV}

The rout recovery mechanism is governed by four configuration parameters. Fig. 1 shows that Snum is the number of hops from the source node to the upstream node before the failure, and Dnum is the number of hops from the upstream node to the destination node. Sneigh and Uneigh are new parameters that are the number of neighbors for the source node and upstream node, respectively. If $(W 1 *$ Snum $)+(W 2 *$ Sneigh $)$ is larger than $(W 3 * D n u m)+(W 4 *$ Uneigh $)$, local repair is done; otherwise, routing begins again from the source node. 


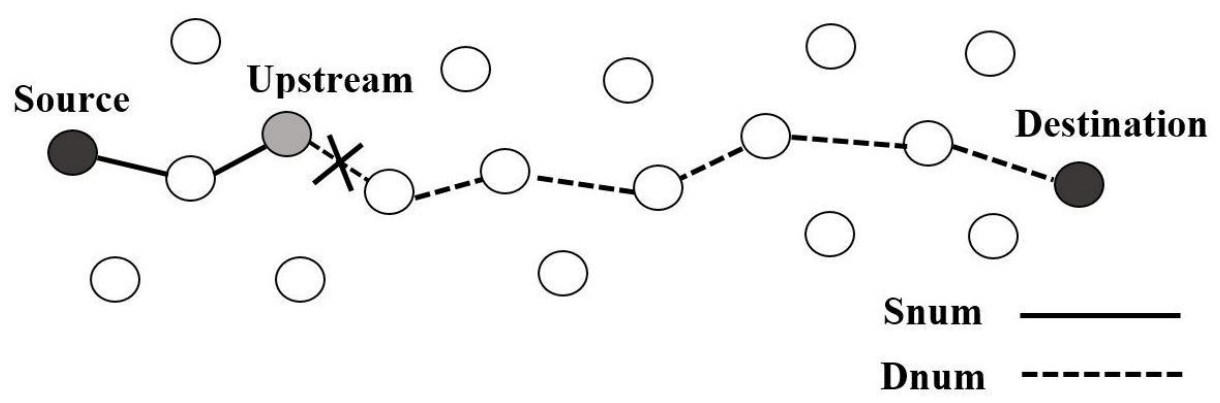

Fig1. Link failure in mobile ad-hoc network

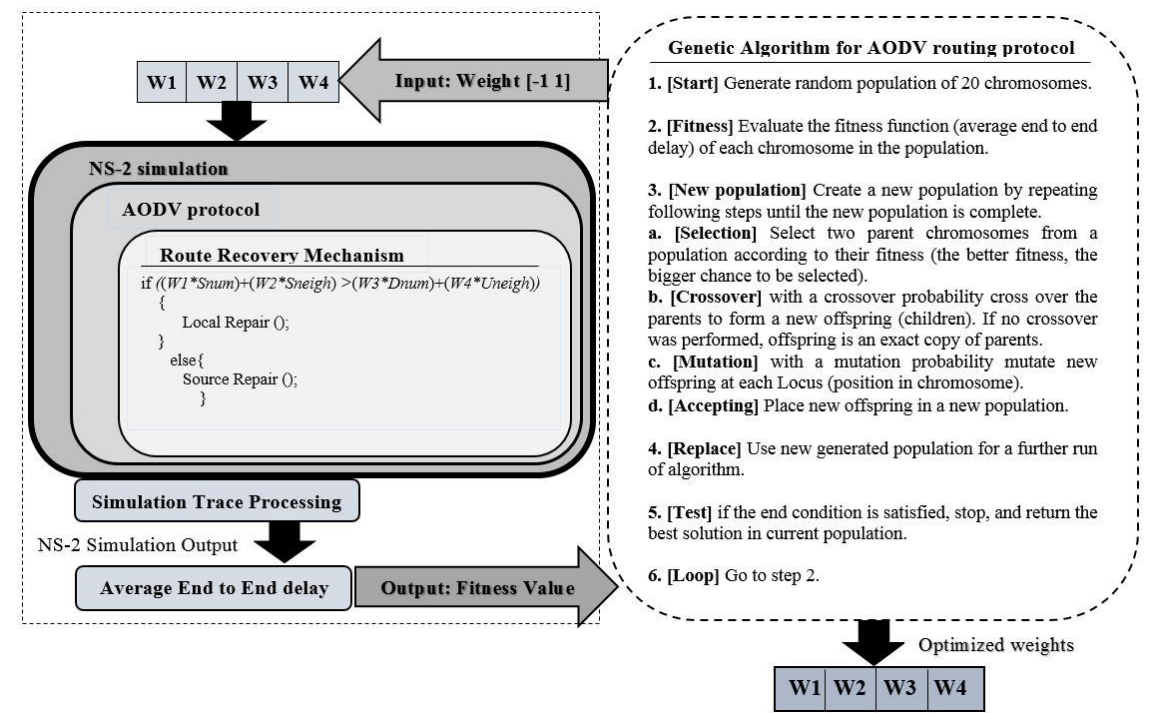

Fig. 2. Optimization strategy for AODV routing protocol in NS2

Weights W1, W2, W3, and W4 are real numbers in the set [-1 1]. The weights are considered to be chromosome genes and are initialized using the GA. Each weight enters the route recovery procedure and returns the value of delay as a fitness value to the GA. This is repeated to obtain the best value for the weight according to the stop circumstances.

In the GA, 20 new populations are first randomly generated and each receives its own fitness value. The children produced by crossover with a probability of 0.8 and by mutation with a probability of 0.25 are transferred to the next generation. After 100 iterations in the GA, 2000 calls are made to the network simulation scenario for delay analysis. The GA then generates the four weights that create the least delay value as output. Fig. 2 summarizes the automatic methodology to find an appropriate combination of weights for the AODV protocol in the MANETs. The proposed method integrates evolutionary search using a GA, routing simulation in the MANETs using the NS-2 network simulator, and a set of scripts developed to evaluate the quality of service using the NS-2 output.

\section{Simulation and Analysis}

The modified AODV scheme was compared with the GAAODV and the AODV and its performance was evaluated. The schemes were evaluated using the following performance metrics:

1. Control overhead: number of control packets transmitted per data packet delivered,

2. Average end-to-end delay: average time taken for all data packets to be transmitted across a network from source to destination,

3. Packet delivery ratio: number of packets that were successfully received divided by the number of packets sent out, 


\subsection{Network simulation}

The simulation used a NS-2 [v-2.35] network simulator. Table 1 lists the simulation parameters. The Mac protocol was based on IEEE 802.11 and the propagation model was a two-ray ground reflection model. The simulation scenario consisted of 60 mobile nodes randomly distributed in a $1000 \mathrm{~m} \times 400$ $\mathrm{m}$ rectangular space. The maximum speed of the nodes was one of six maximum moving speeds $(0$, $5,10,15,20,25 \mathrm{~m} / \mathrm{s})$. The CBR data packets comprised 512 bytes and the sending rate is 4 packets $/ \mathrm{s}$. The transmission range of each node was $250 \mathrm{~m}$.

Simulations were run for $60 \mathrm{~s}$. Each curve point in the results represents an average of 10 runs with identical traffic loads. The confidence level was set to $95 \%(\mathrm{p}=0.05)$; the results were statistically different if they occurred at $\mathrm{p}<0.05$.

\section{Table 1}

Simulation parameters in NS-2

\begin{tabular}{llll}
\hline Parameter & Value & Parameter & Value \\
\hline Simulation time & $60 \mathrm{~s}$ & Propagation model & Two Ray Ground \\
Simulation area & $1000 \mathrm{~m} * 400 \mathrm{~m}$ & Mac protocol & IEEE 802.11 \\
Number of Nodes & 60 & transmission range & $250 \mathrm{~m}$ \\
Node speed & $0,5,10,15,20,25,30 \mathrm{~m} / \mathrm{s}$ & Data payload & 512 bytes \\
\hline
\end{tabular}

\subsection{Performance result}

The ability of GAAODV and the original AODV to change the topology dynamically by changing node mobility through variation of the maximum speed was evaluated.

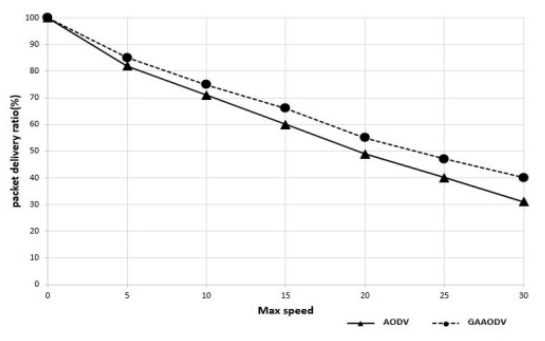

Fig. 3. Comparison of packet delivery ratio

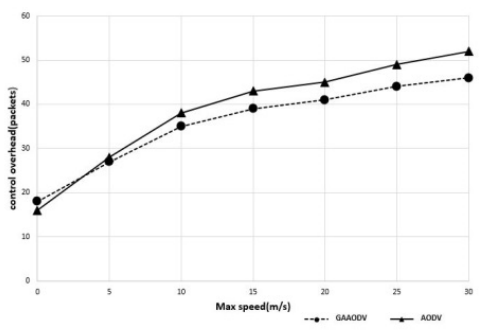

Fig. 4. Comparison of control overhead

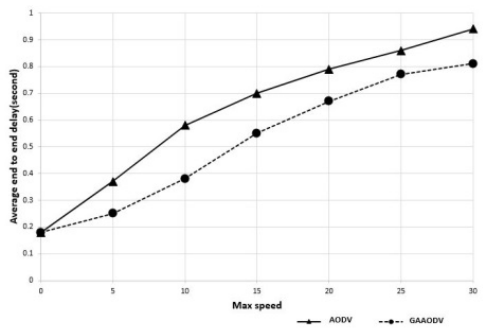

Fig. 5. Comparison of average end to end delay

Fig. 3 compares the packet delivery ratio in which GAAODV represents the proposed AODV-based GA scheme. Simulation results indicate that proposed method outperformed AODV, since AODV does not use a GA, thus, more data loss occurred whenever a link was disrupted along the route. Fig. 4 compares the control overhead. Simulation results indicate that, when nodes stopped or moved slowly, the control overhead of the GAAODV resembled that of the AODV. Increasing the mobile rate decreased the control overhead for GAAODV over that for AODV because it decreased the route recovery using the GA. Fig. 5 compares the average end-to-end delay. The AODV incurred the largest delay because of the frequent route discoveries required in the dynamically changing topology. Since GAAODV utilized GA to find an appropriate combination of weights whenever a link was disrupted along the route, the average end-to-end delay decreased.

\section{Conclusion}

This paper examined the problem of path failure in mobile ad-hoc networks. A new method is proposed to improve the performance of the route recovery mechanism of the AODV protocol. When an active path is broken, the route repairing procedure is responsible for selecting local repair or source repair. The number of neighbors of the candidate nodes and weights were added to the decision function to make better decisions using the GA. Simulation results indicate that the 
GAAODV obtains a lower average end-to-end delay and lower routing overhead than the conventional AODV. Avenues for future study include improving the method used for the automatic search and tackling the weights as a multi-objective problem. Regarding the first issue, the use of new fitness functions should be considered that take into account new QOS metrics, such as the packet delivery ratio and routing overhead. In addition, the approach proposed in this paper could be used by other evolutionary algorithms. Regarding the second issue, the study of explicit multi-objective approaches for the problem is suggested since the packet delivery ratio may vary in inverse proportion with the delay and overhead.

\section{References}

Al-Omari, S. A. K., \& Sumari, P. (2010). An overview of mobiles ad hoc networks for the existing protochols and applications. International Journal on Applications of Graph Theory in Wireless Ad hoc Networks \& Sensor Networks, 2(1).

Bilgin, Z., \& Khan, B. (2010). A dynamic route optimization mechanism for AODV in MANETs. Paper presented at the Communications (ICC), 2010 IEEE International Conference on.

Das, S. R., Belding-Royer, E. M., \& Perkins, C. E. (2003). Ad hoc on-demand distance vector (AODV) routing.

Dong, X., Bailong, L., \& Rubo, Z. (2011). Improved AODV-Based Local Recovery Mechanism in Multiple AUVs System Computing and Intelligent Systems (pp. 47-53): Springer.

Gen, M., \& Cheng, R. (2000). Genetic algorithms and engineering optimization (Vol. 7): John Wiley $\&$ Sons.

Huang, T.-C., Huang, S.-Y., \& Tang, L. (2010). AODV-based backup routing scheme in mobile ad hoc networks. Paper presented at the Communications and Mobile Computing (CMC), 2010 International Conference on.

Liu, H., \& Raychaudhuri, D. (2005). Label switched multi-path forwarding in wireless ad-hoc networks. Paper presented at the Pervasive Computing and Communications Workshops, 2005. PerCom 2005 Workshops. Third IEEE International Conference on.

Marina, M. K., \& Das, S. R. (2001). On-demand multipath distance vector routing in ad hoc networks. Paper presented at the Network Protocols, 2001. Ninth International Conference on.

Mbarushimana, C., \& Shahrabi, A. (2007). Comparative study of reactive and proactive routing protocols performance in mobile ad hoc networks. Paper presented at the Advanced Information Networking and Applications Workshops, 2007, AINAW'07. 21st International Conference on.

Pan, M., Chuang, S.-Y., \& Wang, S.-D. (2005). Local repair mechanisms for on-demand routing in mobile ad hoc networks. Paper presented at the Dependable Computing, 2005. Proceedings. 11th Pacific Rim International Symposium on.

Perkins, C. E., \& Royer, E. M. (1999). Ad-hoc on-demand distance vector routing. Paper presented at the Mobile Computing Systems and Applications, 1999. Proceedings. WMCSA'99. Second IEEE Workshop on.

Rahman, A. H. A., \& Zukarnain, Z. A. (2009). Performance comparison of AODV, DSDV and IDSDV routing protocols in mobile ad hoc networks. European Journal of Scientific Research, 31(4), 566-576.

Sarkar, S. K., Basavaraju, T., \& Puttamadappa, C. (2007). Ad hoc mobile wireless networks: principles, protocols and applications: CRC Press.

Whitley, D. (1994). A genetic algorithm tutorial. Statistics and computing, 4(2), 65-85.

Youn, J.-S., Lee, J.-H., Sung, D.-H., \& Kang, C.-H. (2006). Quick local repair scheme using adaptive promiscuous mode in mobile ad hoc networks. Journal of Networks, 1(1), 1-11.

Yujun, L., \& Lincheng, H. (2010). The research on an AODV-BRL to increase reliability and reduce routing overhead in MANET. Paper presented at the Computer Application and System Modeling (ICCASM), 2010 International Conference on. 